\title{
Home Medication Review to Identify the Medication Errors in the Paediatric Population
}

\author{
A Ramakrishna Shabaraya ${ }^{1}$, Jewel Janice Fernandes ${ }^{2}$, Mohammed Adil Ashraf $^{3}$, \\ Karol Sara Mathew ${ }^{4}$, Akhila Ullas ${ }^{5}$ \\ ${ }^{1}$ Professor and Head, Department of Pharmacy Practice, ${ }^{2,3,4,5}$ Student PharmD, \\ Srinivas College of Pharmacy, Mangalore, Karnataka, India \\ Corresponding Author: Jewel Janice Fernandes
}

\begin{abstract}
Medication errors are therapeutic obstacles that have the potential to threaten the patient outcomes and result in unexpected events. Home medication review (HMR) is a patientfocused process which advocates the optimal and quality use of medication at the patient's home. It involves a systematic and detailed assessment of the patient's medication in order to identify and meet the medication-related needs as well as to identify and prevent medication errors. The aim of this study is to identify the medication errors in paediatric population. The paediatric population is a vulnerable part of the community as they should be treated with utmost care due to their differing pharmacokinetic properties. This study is a community based prospective observational study including 150 paediatric participants. A specially designed data collection form will be used to collect the data from the patients who are eligible for the study. Several dosing and administration errors were also found in the study. Several studies have been done under home medication review but only a few with paediatric population is seen. Home medication review can be very useful for the society if it is implemented. It will be helpful to the community in clarifying any misunderstandings regarding the treatment and can lead to better outcomes.
\end{abstract}

Keywords: Home medication review, paediatric population, adherence.

\section{INTRODUCTION}

Medication errors are defined as any error in drug ordering, transcribing, dispensing, administering or monitoring. Medication errors have the potential to threaten the patient outcomes and result in unexpected events. The paediatric population belongs to the special population that ranges from the age of 0-18 years, i.e.; from infancy to adolescence. Children are particularly vulnerable to medication errors because of their unique physiology and pharmacological differences. Besides this child doses are calculated based on age, weight and body surface area, which is prone to errors. There is also inadequate information on child medications administration and inadequate availability of appropriate dosage forms and concentrations. Children have a faster growth rate compared to adults resulting in weight changes over time and a recalculation of drug doses is required, especially in neonates Dosing errors and administration errors are two of the most commonly observed medication errors in paediatric population due to complex medicines use process. Despite all clinical strategies to avoid mistakes like training doctors, pharmacists and nurses to ensure that they are having complete oversight in administering, dispensing, standardizing and identifying medications effectively, medication errors are yet prevalent. Even though they occur at rates similar to adults 
but have three times the potential to cause harm.

Dosing errors usually are the result of the fact that most of the medications are developed in concentrations appropriate for adults and the paediatric indications and dosing guidelines are often not included. Hence, the dosing requires precise child dose calculations based on various factors. Children are usually administered with liquid dosage forms to ensure easy administration, unlike in solid dosage forms like tablets. This in fact precipitates various administration errors due to inconsistency. Children are rarely undergoing clinical trials for various preparations as they belong to the special population; this makes therapy even trickier. Since medication errors cause significant morbidity and mortality in the paediatric population there is a greater need for preventive measures and close monitoring. A complete knowledge of the paediatric pharmacokinetics and stage of development can aid in the accurate prescription of medication for children and minimizing medication errors. Promoting safe and efficacious use of drugs in children to ensure a good therapeutic outcome is of paramount importance.

This research explores the possible reasons for dosing and administration errors in the paediatric population using home medication review of the medications received by children in different households. Home medication review (HMR) is a patient-focused process which advocates the optimal and quality use of medication at the patient's home. It involves systematic assessment of the patient's medication in order to identify and meet the medicationrelated needs as well as to identify and prevent medication errors. HMR can be a powerful tool in preventing medication errors and promoting safe drug use.

\section{MATERIALS \& METHODS}

Study site: A prospective observational study was carried out within Dakshina Kannada District, Karnataka.
Study Design: Community based prospective observational study.

Study period: The study was conducted for duration of 6 months from August 2019 to February 2020.

Ethical clearance: The study protocol was approved by the Institutional Ethics Committee (IEC) of in Srinivas Institute of Medical Science and Research Centre, Mukka, Mangalore

\section{Study criteria: \\ Inclusion criteria:-}

- Patients of age 18 years and below

- Patient on medication for any illness (current or chronic)

\section{Exclusion criteria:-}

- Patients of age above 18 years

- Patients who are homeless or do not have a proper place to stay

Source of data: The data for study was taken from the patient's caregiver/parents that consist of prescriptions, case files, various test reports, medication formulations which are present at home that are used by the paediatric population.

Sampling method: Various houses in different places of Dakshina Kannada with paediatric population who meet the required criteria were visited between September 2019 and February 2020. A total of 150 study subjects were included in the study which may include more than one subject from the same household who satisfy the selection criteria.

Study method: A specially designed data collection form was used to collect the data from the patients who are eligible for the study. Data collected included demographic details, presenting complaints, medical \& medication history, diagnosis, treatment including dose, frequency, formulation, duration and patient progress. All the details were kept confidential.

Data Analysis: The collected data was analysed using Microsoft Excel 2010. The filters were used to separate the different 
groups of the study population for scrutiny of the study.

\section{Operational modality:}

$\square$ Ethical committee approval

Preparation of Data collection form

Community visit and selection of subjects

Signature of informed consent

Data collection

Analysing data using Microsoft Excel

$\square$ Presentation of data with tables and graphs

\section{RESULT \\ DEMOGRAPHIC DETAILS OF THE STUDY POPULATION}

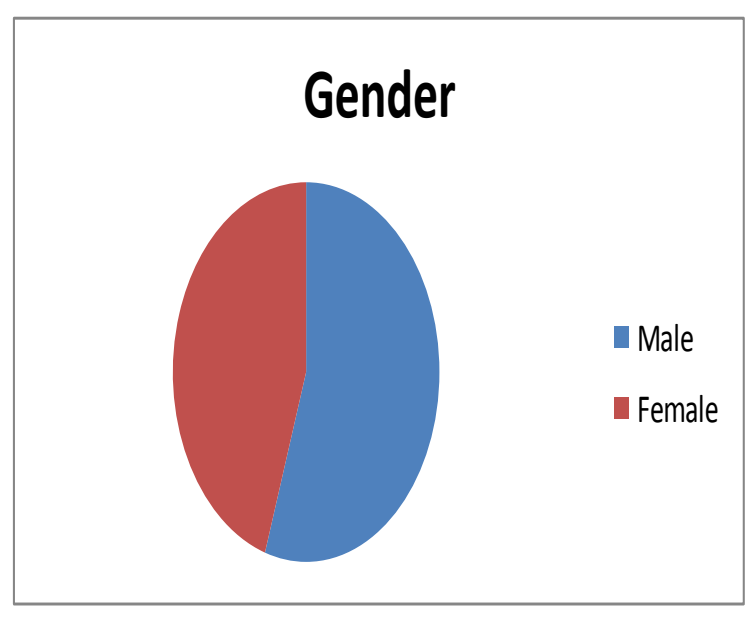

Figure 1: Gender of the study participants

The study was carried out in about 150 paediatric patients in the community level acquiring information from the patients or/and from the caregiver/parents. Among which 82 were male and 68 were female subjects. Here figure 1 gives the percentage of male and female participants in the study. Among the paediatric patients participated in the study 55 subjects were between the age group 0-5 years, 53 subjects were between the age group 6-10 and 42 between 11-18 years of age. When BMI of the subjects are considered 32 subjects were found between 10.1- 15, 94 between 15.1-20 and 24 from 20.1 and above. Out of 150 subjects 111 subjects were found to be underweight with a BMI less than 18.5 while 38 were of ideal BMI and only 1 subject was overweight. Table 1 gives the demographic features of the participants of the study.

Table 1: Demographic features of the study participants

\begin{tabular}{|l|ll|l|}
\hline GENDER & $\bullet$ & Male & $82(55 \%)$ \\
& $\bullet$ & Female & $68(45 \%)$ \\
\hline AGE & $\bullet$ & 5 years & $55(36.6 \%)$ \\
& $\bullet$ & 6-10 years & $53(35.3 \%)$ \\
& $\bullet$ & $11-18$ years & $42(28 \%)$ \\
\hline BMI & $\bullet$ & $10.1-15$ & $32(21.3 \%)$ \\
& $\bullet$ & $15.1-20$ & $94(62.6 \%)$ \\
& $\bullet$ & 20.1 and above & $24(16 \%)$ \\
\hline
\end{tabular}

Medication related problems like administration errors and dosing errors are identified from the information given by the patients / caregiver. Since majority of the pediatric population are prescribed oral liquid formulation like syrups, suspensions, devices for administration of such formulations were checked which were devices supplied with medicine such as dosing cup or household measures such as teaspoon, tablespoon. It was found that out of 130 pediatric patients who used oral liquids 101 patients used devices supplied with medicine and 29 used household measures. Among the patients who used household measures it was seen that 17 were aware and 12 were unaware about the teaspoon measurement $(1$ teaspoon $=5 \mathrm{ml})$ whereas 16 were aware and 13 were unaware about the tablespoon measurement (1 tablespoon $=15 \mathrm{ml})$. These errors can contribute to both dosing and administration errors. The other parameters which were checked are the patient's / caregiver's knowledge regarding $\mathrm{BD}$ dosage or twice a day dosing interval, before meal dosing duration, after meal dosing duration. It was seen that about 77 study participants had the right knowledge about $\mathrm{BD}$ dosing while 73 did not have the correct knowledge about BD dosing interval. The knowledge about before meal and after meal dosing duration were also checked and was found that 114 people had the right knowledge about before meal duration while 36 did not and about after meal 88 had right knowledge and 62 did not. 
A Ramakrishna Shabaraya et.al. Home medication review to identify the medication errors in the paediatric population.

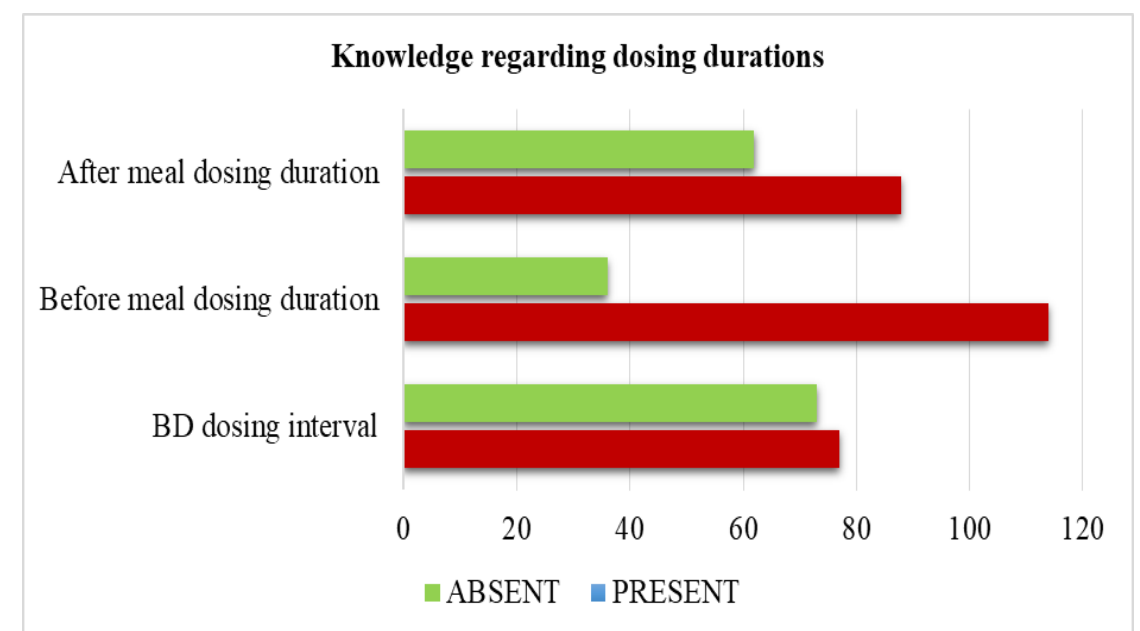

Fig 2: Knowledge regarding dosing durations

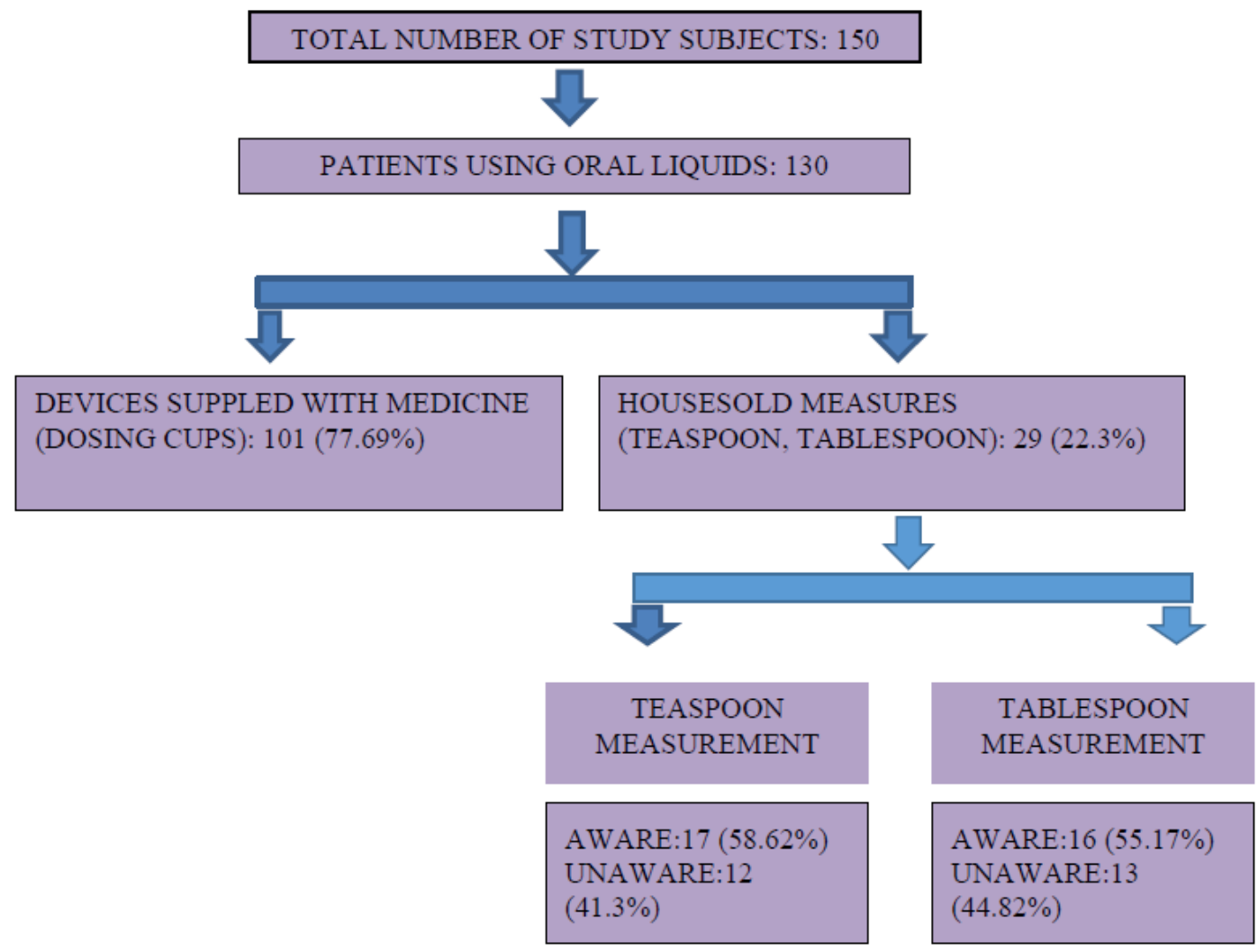

Fig 3: Knowledge regarding drug dosage administered using household devices

\section{DISCUSSION}

Pediatric medication is usually consisting of drugs for ailments like cold, fever, allergy, nausea and vomiting. These drugs are administered whenever necessary and therefore medication related problems like administration errors and dosing errors were observed in the study. Most of the pediatric medication was observed to be given in the form of oral liquid dosage like syrups, drops, emulsions and suspensions. These preparations have the highest chances of dosing errors and variations as it is not given in the unit dosage form. Therapeutic errors among the children in the community mainly involve very young children, parents administering single medications in the home, dosing errors and OTC medications. Also the devices used to measure and administer these preparations varied from household to household. It was found that most of the parents/caregivers used the devices provided with the medication, e.g. the measuring cup. Only a few number of 
individuals preferred household devices like spoons to administer the drug. Among those who used these household devices, a fair number of subjects were not aware of the accurate dose that would be administered using a household device. Resulting in both administration and dosing errors.

From the study it was also found that the number of people having the right knowledge regarding the $\mathrm{BD}$ dosage and interval and unaware of the same were nearly equal. This proves that there is still lack of complete knowledge regarding the dosage interval and its importance on the efficacy of a treatment plan. The study also focused on the information the subjects had on the before meal and after meal duration and it was concluded that most of the subjects had the right information but a fair number failed to provide the right information (Figure 1). Drugs require the right gastric environment to work to its fullest potential and result in the expected therapeutic results. These parental errors in dosing liquid medication can be decreased through the right education.

\section{CONCLUSION}

Dosing and administration errors were found to be marginally high in the pediatric population in this study. Since children are administered generally with oral dosage forms like syrups the dosing errors tend to be higher compared to unit solid dosages like tablets in the adults. Medication errors in the pediatric patients can lead to failure or insufficient achievement in therapeutic goals. Children need special attention and awareness in therapy as they need special dosing considerations and close monitoring as their bodies are in different stages of development compared to that of adults. There are numerous sources of medication errors in the pediatric population but every adversity has a suitable solution, either to prevent it or solve it. It was found that something minimal like the knowledge of before and after meal dosing time, dosing interval and household dosing appliances can result in a wide array of medication errors, including both dosing and administration errors. The fact that children are not usually available for patient counseling increases the chance of errors. It's essential to realize that errors are always possible and all the necessary steps required to prevent this must be undertaken to ensure safe and efficacious therapy in the pediatric population. The errors can result from the end of the medical professionals or the patient/parent. Dosing and administration errors in children are the most prevalent errors with a bigger chance of resulting in morbidity and mortality. Lack of complete knowledge about the therapy from the patient's/caretaker's end is still a major setback in successful therapy. Communication is the key to achieve efficient results in pediatric therapy. Something as simple as detailed and systematic patient and parent counseling regarding proper drug administration and dosing has the capacity to prevent adverse events and achieve the expected therapeutic results and promoting safe and efficacious drug therapy in children. This study helps to bring to light the various loose-ends with regard to medication errors in pediatric therapy and resolving them before-hand.

\section{Acknowledgement: None}

Conflict of Interest: None

\section{Source of Funding: None}

\section{Ethical Approval: Approved}

\section{REFERENCES}

1. Ghaleb MA, Barber N, Franklin BD, Yeung VW, Khaki ZF, Wong IC. Systematic review of medication errors in pediatric patients. Annals of Pharmacotherapy. 2006 Oct;40(10):1766-76.

2. Stratton KM, Blegen MA, Pepper G, Vaughn T. Reporting of medication errors by pediatric nurses. Journal of pediatric nursing. 2004 Dec 1;19(6):385-92.

3. Kozer E, Scolnik D, Macpherson A, Keays T, Shi K, Luk T, Koren G. Variables 

population.

associated with medication errors in pediatric emergency medicine. Pediatrics. 2002 Oct 1;110(4):737-42.

4. Otero P, Leyton A, Mariani G, Cernadas JM. Medication errors in pediatric inpatients: prevalence and results of a prevention program. Pediatrics. 2008 Sep $1 ; 122(3): \mathrm{e} 737-43$.

5. Fortescue EB, Kaushal R, Landrigan CP, McKenna KJ, Clapp MD, Federico F, Goldmann DA, Bates DW. Prioritizing strategies for preventing medication errors and adverse drug events in pediatric inpatients. Pediatrics. 2003 Apr 1;111(4):722-9.

6. Cimino MA, Kirschbaum MS, Brodsky L, Shaha SH, Child Health Accountability Initiative. Assessing medication prescribing errors in pediatric intensive care units. Pediatric critical care medicine. 2004 Mar 1;5(2):124-32.

7. Kaushal R, Bates DW, Abramson EL, Soukup JR, Goldmann DA. Unit-based clinical pharmacists' prevention of serious medication errors in pediatric inpatients. American Journal of Health-System Pharmacy. 2008 Jul 1;65(13):1254-60.

8. Larsen GY, Parker HB, Cash J, O'Connell M, Grant MC. Standard drug concentrations and smart-pump technology reduce continuous-medication-infusion errors in pediatric patients. Pediatrics. $2005 \mathrm{Jul}$ $1 ; 116(1): e 21-5$.

9. Kaufmann J, Laschat M, Wappler F. Medication errors in pediatric emergencies: a systematic analysis. Deutsches Ärzteblatt International. 2012 Sep;109(38):609.

How to cite this article: A Ramakrishna Shabaraya, Fernandes JJ, Ashraf MA et.al. Home medication review to identify the medication errors in the paediatric population. International Journal of Research and Review. 2021; 8(7): 462-467. DOI: https://doi.org/10. 52403/ijrr.20210765 